\title{
Nonlinear-Dispersive GaAs FET Drain-Current Model for Harmonic Balance Simulation.
}

\author{
KW Eccleston \\ Department of Electrical Engineering \\ National University of Singapore \\ SINGAPORE 119260
}

\begin{abstract}
Popular GaAs FET large-signal drain conduction current models depend only on instantaneous terminal voltages, and ignore important phenomena that result in low frequency dispersion. To be valid at microwave frequencies, both the dc and time-varying components of current must be accurately modelled. This paper proposes a GaAs FET drain current model, which includes rate-dependent body and thermal effects, and therefore has the capability to accurately predict both the dc and time-varying components of drain current. Further, this model is particularly suited to harmonic balance simulation of microwave circuits.
\end{abstract}

\section{INTRODUCTION}

The accuracy of the GaAs FET large-signal drain conduction current model has a significant effect on the accuracy of large-signal simulations of microwave circuits. It is well known that the drain conduction current is both voltage and temperature dependent. Temperature dependence is important as power dissipated by the FET, along with finite thermal resistance, results in significant channel heating.

Many of the physical processes that govern the drain conduction current (eg. body effect and surface states) have time-constants of order milliseconds and can only track slowly varying voltages. Likewise, due to significant thermal mass, the thermal time-constant is of the order of milliseconds and hence the channel temperature can only track a slowly varying power dissipation. These rate-dependent phenomena manifest themselves as the so called low frequency drain current dispersion. In the context of operation at microwave frequencies: the channel temperature can only respond to the dc power dissipation; whilst the body effect consists of a component that can only respond to dc voltages, and another to rf voltages.

In summary, for microwave circuits, the FET drain current may be written as a function of the instantaneous FET terminal voltages, their timeaverages, and the time-average FET power dissipation. Hence the waveforms of the FET terminal voltages and the instantaneous power dissipation must be considered.

Popular drain current models described in the literature [1][2][3] are dependent only on instantaneous terminal voltages, and as they are derived from dc measurements, they fail to account for the low frequency dispersion, thereby resulting in modelling inaccuracy at RF and microwave frequencies. To resolve this problem, it has been proposed that the drain current nonlinearity parameters should be either extracted from pulsed I/V measurements [4] or large-signal RF measurements [5]. However, models derived in this manner will fail to accurately predict the dc drain current, hence dc power dissipation.

Recent microwave FET models [6]-[8], which have concentrated on accurate prediction of both the rf and dc components of drain current, do not explicitly account for ratedependent effects. Rather, they essentially resolve the drain current into two components: a dc model based on dc measurements, and an RF model derived from the bias behaviour of smallsignal $g_{m}$ and $g_{o}$ obtained from microwave $S$ parameters at numerous bias points. The models 
by Rizzoli et al [6] and Filicori et al [7] are temperature dependent. In the case of Rizzoli et al [6], the temperature is assumed time dependent and hence is aimed at simultaneous electro-thermal simulation. On the other hand, Filicori et al [7] calculate the time-average power dissipation. Kompa [8] does not explicitly account for thermal effects. Parker [9] proposes a model, that explicitly models rate-dependent thermal and body effects. However, Parker's model is aimed at large-signal SPICE simulation requiring calculations to begin from circuit startup, and hence is inefficient for large-signal steady-state simulation.

It is therefore the purpose of this paper to propose a dispersive drain current model which provides accurate predictions of both the dc and time-varying components of the drain current at microwave frequencies. The features of the proposed model are: highly suited for harmonic balance simulators, is based on a popular drain current models, and rate-dependent effects are explicitly accounted for.

\section{DRAIN CURRENT MODEL}

It is assumed that the waveforms $V_{G S}(t)$ and $\mathrm{V}_{\mathrm{DS}}(\mathrm{t})$ are both periodic and their periods are small compared to the time constants associated with the body effect and self-heating. Thus the model will only be valid at $\mathrm{dc}$ and at RF frequencies above say $1 \mathrm{MHz}$, which is sufficient for most RF and microwave applications. For the sake of convenience, let the vector $\mathrm{V}(\mathrm{t})$ equal $\left[\mathrm{V}_{\mathrm{GS}}(\mathrm{t}), \mathrm{V}_{\mathrm{DS}}(\mathrm{t})\right]^{\mathrm{T}}$ and the vector $\mathrm{V}_{0}$ equal $\left[V_{G S 0}, V_{D S 0}\right]^{T}$, where $V_{G S 0}$ and $V_{D S 0}$ are $d c$ components (time-averages) of $V_{G S}(t)$ and $V_{D S}(t)$ respectively.

The rate-dependent body effect is based on Parker's body effect model [9]:

$$
\begin{aligned}
\mathrm{V}_{\mathrm{T}}\left(\mathbf{V}(\mathrm{t}), \mathrm{V}_{0}\right) & =\mathrm{V}_{\mathrm{TO}}+\gamma_{\mathrm{LF}}\left(\mathrm{V}_{\mathrm{DSO} 0}-\mathrm{V}_{\mathrm{GSO}}\right) \\
& +\gamma_{\mathrm{HF}}\left(\mathrm{V}_{\mathrm{DS}}(\mathrm{t})-\mathrm{V}_{\mathrm{GS}}(\mathrm{t})-\mathrm{V}_{\mathrm{DSO}}+\mathrm{V}_{\mathrm{GSO}}\right)
\end{aligned}
$$

where both the second and third terms can respond to the dc bias. Only the third term can respond to rf voltages and is zero under static conditions.

The isothermal drain current model is based on the Statz transfer characteristic [3], and the Parker subthreshold model [9]:

$$
\begin{aligned}
& I_{\text {DSies }}\left(V(t), v_{0}\right) \\
& =\frac{\beta\left[v_{0}\left(V_{(t)}, v_{0}\right)\right]^{2}}{1+b v_{0}\left(v_{(t)}, v_{0}\right)} \tanh \left(\alpha V_{D S}(t)\right)\left(1+\lambda v_{D S}(t)\right)
\end{aligned}
$$

where:

$$
\begin{aligned}
& \mathrm{v}_{0}\left(\mathbf{v}(t), \mathbf{v}_{\bullet}\right) \\
& =\sigma \ln \left(1+\exp \left(\frac{\mathrm{v}_{\mathrm{Gs}}(\mathrm{t})-\mathrm{v}_{\mathrm{T}}\left(\mathbf{v}(t), \mathbf{v}_{0}\right)}{\sigma}\right)\right)
\end{aligned}
$$

and the model constants $\alpha, \beta, \lambda$, and $b$ are described by Statz et al [3], and $\sigma$ is described by Parker [9]. This model is only valid when the channel temperature is maintained at absolute ambient temperature, $T_{0}$.

As stated earlier, the channel temperature can only track the time-average power dissipation which is given as the product of the time-averages of both the drain voltage and drain current. The most significant consequence of channel heating in GaAs FETs is decrease of the electron saturation velocity, which will in turn cause the saturation drain current to decrease. Under normal operating temperatures, the saturation velocity in GaAs is approximately inversely proportional to absolute temperature [10]. Assuming that the channel temperature is proportional to the time-average dissipated power:

$$
I_{D S}\left(V(t), v_{0}, I_{D S 0}\right)=\frac{I_{D S i s o}\left(v(t), v_{0}\right)}{1+\delta v_{D S 0} I_{D S 0}}
$$

where $\delta$ is the thermal constant of the FET and $\mathrm{I}_{\mathrm{DS} 0}$ is the dc component (time-average) of $\mathrm{I}_{\mathrm{DS}}$. 
It can be shown, that $\delta$ is given by $R_{t h} / T_{o}$ where $R_{t}$ is the thermal resistance from the channel to ambient. This equation is similar to that used by Parker [9] except that the dc power dissipation is given by $\mathrm{V}_{\mathrm{DSO}} \mathrm{I}_{\mathrm{DS} 0}$.

Equation 3 is implicit as the time average of $I_{D s}$ is required. However, in the steady-state regime under periodic excitation, one need only calculate time averages over one cycle. The steady-state time average of equation 3 gives $\mathrm{I}_{\mathrm{DS} 0}$ :

$$
I_{D S 0}=\left\langle I_{D S}\left(\mathbf{V}(t), v_{0}, I_{D S O}\right)\right\rangle
$$

where the time-average is calculated over one period of $\mathbf{V}(\mathrm{t})$. Substituting into equation 3 , and noting that the denominator of equation 3 is constant under steady-state conditions:

$$
I_{D S 0}=\frac{\left\langle I_{D S i b 0}\left(\mathbf{v}(t), \mathbf{v}_{0}\right)\right\rangle}{1+\delta v_{\text {DSO }} I_{D S O}}
$$

of which the solution for $\mathrm{I}_{\mathrm{DS} 0}$ is:

$$
\begin{aligned}
& I_{D S 0}\left(\mathbf{V}(t), \mathbf{v}_{0}\right) \\
& =\frac{-1+\sqrt{1+48 V_{\text {DSO }}\left\langle I_{\text {DSieo }}\left(\mathbf{v}(t), v_{0}\right)\right\rangle}}{28 V_{\text {DSO }}}
\end{aligned}
$$

Clearly, $I_{\mathrm{DS} 0}$ is both voltage and voltage waveform dependent. In a harmonic balance implementation, $\mathrm{I}_{\mathrm{DSiso}}$ is first calculated at each time step over ONE cycle, then its time average and hence $I_{D s 0}$ is calculated, finally $I_{D S}$ is calculated at each time step by dividing $\mathrm{I}_{\mathrm{DSiso}}$ at each time step by the term $1+\delta V_{D S 0} I_{D S 0}$.

For completeness, it follows in a similar manner, that with NO rf excitation present, the $\mathrm{dc}$ drain current is given by:

$$
\begin{aligned}
& I_{\text {DSdo }}\left(v_{0}\right) \\
& =\frac{-1+\sqrt{1+48 v_{\text {DSO }} I_{\text {DSieo }}\left(v_{0}, v_{0}\right)}}{2 \delta v_{\text {DSO }}}
\end{aligned}
$$

and will in general be different from $\mathrm{I}_{\mathrm{DS} 0}$ for a given value of $\mathbf{V}_{0}$.

\section{NUMERICAL EXXAMPLE}

To illustrate the behaviour of the proposed model, the drain current was calculated and plotted for the following parameter values: $\alpha$ $=2, \beta=0.03, b=0.2, \gamma_{\mathrm{LF}}=-0.01, \gamma_{\mathrm{HF}}=-0.08$, $\mathrm{V}_{\mathrm{T} 0}=-1.9, \lambda=0.001, \sigma=0.1$ and $\delta=0.15$ (non-isothermal case). Figure 1 and 2 demonstrate the behaviour of the model for the isothermal and non-isothermal cases respectively. Superimposed on Figures 1 and 2 are the dynamic operating locus under class $B$ operation $(-x-$ line) with the gate voltage equal to $-1.9+2.3 \cos (\omega t)$, the drain bias voltage equal to $5 \mathrm{~V}$, and the load impedance is $115+\mathrm{j} 0 \Omega$ at the fundamental, and zero at dc and all other harmonics.

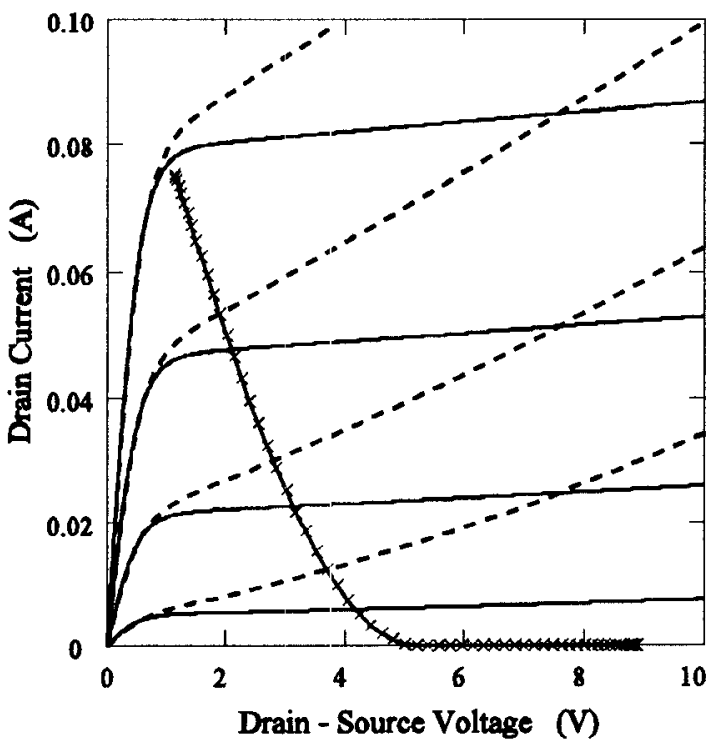

Figure 1. Isothermal drain current behaviour: $\mathrm{DC}\left(\mathrm{V}_{\mathrm{GS}}=0,-0.5,-1.0,-1.5 \mathrm{~V}\right)$ - solid lines, Pulsed dashed lines $\left(\mathrm{V}_{\mathrm{GS}}=0,-0.5,-1.0,-1.5 \mathrm{~V}\right)$, Class $\mathrm{B}$ operating locus ( $-\mathrm{x}-\mathrm{)}$.

Comparing the $\mathrm{dc} \mathrm{i} / \mathrm{v}$ characteristics (solid lines), the effect of a non-zero thermal resistance on the saturation region dc output conductance becomes apparent. In particular, depending on the value of thermal resistance, the de output conductance can be negative as indicated in Figure 2 . The pulsed $\mathrm{j} / \mathrm{v}$ characteristics (dashed lines) in both the iso-thermal or non-iso-thermal cases are virtually identical as the duty cycle is 
small, thus power dissipated, and hence temperature rise, are small. Therefore the pulsed $\mathrm{i} / \mathrm{v}$ characteristics predominantly reveals the ratedependent body effect.

It is interesting to note that the class B operating loci of the two cases differs only slightly, and again results from low average dissipated power under this condition. Such results would suggest that the rate-dependent body effect is more important than thermal effects in class B amplifiers. On the other hand, in class A amplifiers, due to higher average power dissipation, the operating loci could indeed show significant thermal effects.

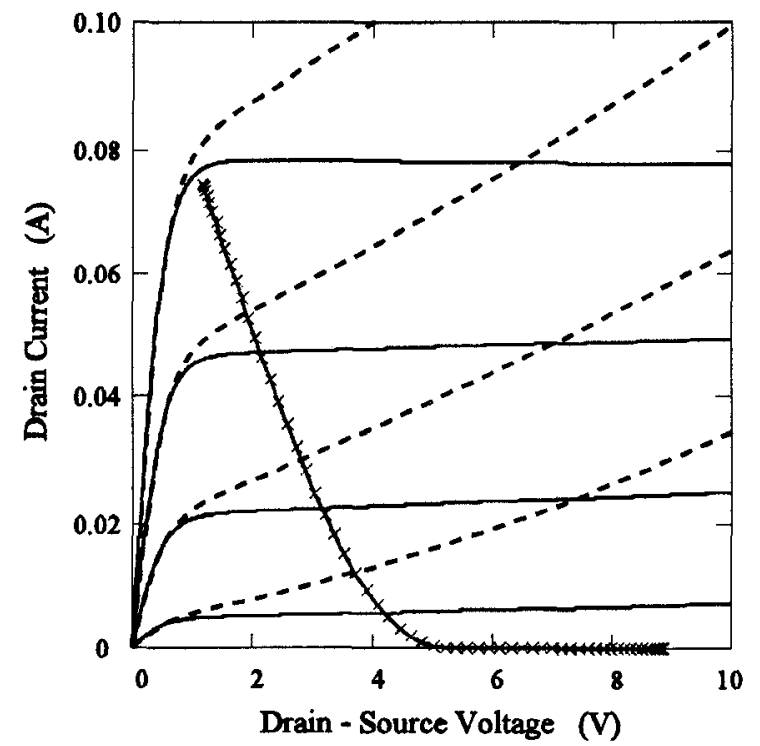

Figure 2. Non-isothermal drain current behaviour: DC $\left(\mathrm{V}_{\mathrm{GS}}=0,-0.5,-1.0,-1.5 \mathrm{~V}\right)$ - solid lines, Pulsed - dashed lines $\left(V_{\mathrm{GS}}=0,-0.5,-1.0,-1.5 \mathrm{~V}\right)$, Class $\mathrm{B}$ operating locus $(-\mathrm{x}-)$.

\section{CONCLUSION}

This paper has proposed a GaAs FET drain conduction current model particularly suited for harmonic balance simulation of microwave circuits. This model explicitly accounts for ratedependent thermal and body effects, and is based on a popular drain current model. It is envisaged that other popular drain current models may be modified in a similar manner.

\section{REFERENCES:}

[1] A. Materka \& T. Kacprzak, "Computer calculation of large signal GaAs FET amplifier characteristics", IEEE T. on Microwave Theory Techniques, Vol. 33, No. 2, Feb. 1985, pp129-135.

[2] W. Curtice \& M. Ettenberg, "A nonlinear GaAs FET model for use in the design of output circuits for power amplifiers", IEEE T. on Microwave Theory Techniques, Vol. 33, No. 12, Dec. 1985, pp13831393.

[3] H. Statz et al, "GaAs FET device and circuit simulation in SPICE", IEEE T. on Electron Devices, Vol. 34, No. 2, Feb. 1987, pp160-167.

[4] M. Paggi et al, " Nonlinear GaAs MESFET Modelling Using Pulsed Gate Measurements ", IEEE T. on Microwave Theory Techniques, Vol. 36, No. 12, Dec. 1988, pp 1593 - 1597.

[5] M. A. Smith et al, "RF Nonlinear Device Characterisation Yields Improved Modelling Accuracy ", 1986 Int. Microwave Symp., 1986 MTTS Digest, pp 381 - 384.

[6] V. Rizzoli et al, "An Electrothermal Functional Model of the Microwave FET Suitable for Nonlinear Simulation", Int. J. of Microwave and MillimeterWave Computer-Aided Engineering, Vol. 5, No. 2, 1995, pp $104-121$.

[7] Filicori et al, 'Empirical Modelling of Low-Frequency Dispersive Effects Due to Traps and Thermal Phenomena in III-IV FET"s", IEEE T. on Microwave Theory Techniques, Vol. 43, No. 12, Dec. 1995. pp2972 - 2981

[8] G. Kompa, "Modelling of Dispersive Microwave FET Devices Using a Quasi-Static Approach", Int. J. of Microwave and Millimeter-Wave Computer-Aided Engineering, Vol. 5, No. 3, 1995, pp 173 -194.

[9] A. E. Parker, "Implementing high-order continuity and rate dependence in SPICE models", IEE Proc.Pt. G, Vol. 141, No. 4, August 1994, pp251 - 257.

[10] S. M. Sze, Physics of Semiconductor Devices, 2nd Ed. John Wiley 1981, pp 44 - 49. 